Copyright by the American Physical Society. Lee, T. K.; Chang, L. N., "Energies of the staggered flux phase: A numerical study," Phys. Rev. B 42, 8720 (R) DOI: http://dx.doi.org/10.1103/ PhysRevB. 42.8720

\title{
Energies of the staggered flux phase: A numerical study
}

\author{
T. K. Lee and L. N. Chang \\ Department of Physics, Virginia Polytechnic Institute and State University, Blacksburg, Virginia 24061
}

(Received 20 June 1990)

\begin{abstract}
Kinetic and magnetic energies of the staggered flux phase, with a fictitious flux of equal magnitude but opposite direction in adjacent square plaquettes, and calculated for the two-dimensional $t-J$ model using the variational Monte Carlo method. They are compared to the energies of the resonating-valence-bond state, the flux phase with half a quantum per plaquette, and the projected Fermi-liquid state. For about $10 \%$ hole concentration the staggered flux phase has the lowest energy of the nonsuperconducting states but its energy is still higher than that of the superconducting $d$-wave state.
\end{abstract}

It is now generally accepted that the large- $U$ Hubbard model (frequently referred to as the $t-J$ model) ${ }^{1,2}$ for a two-dimensional square lattice serves as a good starting point to formulate a description of the high-temperature superconductors. The ground state for this model at half filling, where there is only one electron per site, is by now fairly well understood. It turns out that there are several equivalent ways of describing this ground state, all of which become distinct when charge carriers are introduced by doping. For example, Anderson and coworkers $^{1,3}$ propose the resonating-valence-bond (RVB) state as the ground state for this model. Numerical studies $^{4}$ have indeed demonstrated that at half filling, the RVB state has an energy very close to the long-rangeordered antiferromagnetic (AFM) "ground" state. It is possible to modify the RVB state slightly to become AFM ordered. $^{5}$ For a very small concentration of holes the RVB state becomes more stable than the AFM state.

Alternatively, Affleck and Marston ${ }^{6}$ (AM) propose the flux phase for the ground state of the $t-J$ model. At half filling, the tightly bound electrons are moving under a magnetic flux of half a quantum $\Phi_{0}$ per plaquett. In fact, the flux phase can be shown to be equivalent to the RVB state. ${ }^{7}$ The gap parameter in the RVB state is related to the magnitude of the flux. Away from half filling, however, the relationship between the two becomes unclear.

The $t-J$ model Hamiltonian is manifestly invariant under time-reversal transformation, and at half filling, both of these wave functions respect this symmetry. But away from half filling, while the RVB state would retain this symmetry, by its very nature, the extension of the AM state is expected to break time-reversal symmetry. Since the RVB state has a BCS-like wave function, it is expected to become superconducting ${ }^{4}$ when there are doped charge carriers. The extension of the AM state may be a good description for the normal state away from half filling.

There are at least two possible ways to generalize the AM state away from half filling. The similarity of the AM state to the states that occur in the Hofstadter problem of the motion of an electron in a magnetic field of commensurate flux has prompted the proposal of the commensurate flux phase ${ }^{8}$ (CFP) for the $t-J$ model away from half filling. The CFP is closely related to the anyon state proposed by several groups. ${ }^{9}$ Another possibility is to break the two-dimensional lattice up into two neighboring plaquettes $(A, B)$, each of which encloses an equal but oppositely directed flux. This is called the staggered flux phase (SFP). ${ }^{10,11}$ If the flux is not a half-integral or integral multiple of the basic quantum unit, time-reversal symmetry will be broken.

Recently Liang and Trivedi ${ }^{12}$ showed numerically that in the presence of a finite amount of holes only for $t<J$, the CFP has lower energy than the projected Fermi-liquid state. Hence it becomes important to find out whether the SFP has sufficiently lower energy to be used as a basis for studying normal-state properties. Intuitively, the SFP is expected to have better kinetic energy than the CFP. At a given hole concentration the magnitude of the flux is a variational parameter that varies between the CFP state with half a flux per plaquette and the projected Fermiliquid state with no flux. The Fermi-liquid state has $20 \%$ lower kinetic energy than the CFP. ${ }^{12}$ Further interest in the SFP is enhanced by the result of Zhang's work ${ }^{10}$ showing that SFP is unstable with respect to $d$-wave superconductivity.

To determine whether SFP is a reasonable normal state of the $t-J$ model, we need to evaluate its energy as compared to other states. In order to take into account the condition of rigorous exclusion of double occupancy at the same site, we shall use the numerical variational Monte Carlo method.

The $t-J$ Hamiltonian we shall consider has two parts, the kinetic term $H_{t}$ and the magnetic term $H_{s}$,

$$
H_{t}=-t \sum_{\langle i j\rangle_{\sigma}} P_{d} C_{i \sigma}^{\dagger} C_{j \sigma} P_{d}+\text { H.c. }
$$

and

$$
H_{s}=J \sum_{\langle i j\rangle} \mathbf{S}_{i} \cdot \mathbf{S}_{j}
$$

where $\langle i j\rangle$ is for nearest-neighbor pairs and $P_{d}$ is the Gutzwiller projection operator that excludes double occupancy.

Following Poilblanc, ${ }^{7}$ the wave function of the SFP is constructed by diagonalizing the effective Hamiltonian

$$
H_{0}=-\sum_{\langle i j\rangle_{\sigma}} e^{i a_{i j}} C_{i \sigma}^{\dagger} C_{j \sigma}+\text { H.c. , }
$$


where the phase $a_{i j}$ is chosen such that the total number of phases around the $A(B)$ site plaquette is $2 \pi \Phi$ $(-2 \pi \Phi) . \Phi$ is in units of basic quantum flux $\Phi_{0}$. Given the flux there is still the choice of gauge. The wave function constructed below and the calculated kinetic energy will be gauge dependent. Similar situations occur in the CFP. Readers are referred to Ref. 12 for extensive discussion. It turns out that the lowest energy is obtained by simply taking all $\left|a_{i j}\right|=\pi \Phi / 2$, with $A$ and $B$ sites having opposite sign. The Hamiltonian is easily diagonalized and its quasiparticles are

$$
\tilde{C}_{\mathbf{k} \sigma}=u_{\mathbf{k}} C_{\mathbf{k} \sigma}+v_{\mathbf{k}} C_{\mathbf{k}}+\mathbf{Q} \sigma
$$

where the wave vector $Q=(\pi, \pi)$,

$$
\left|u_{\mathbf{k}}\right|^{2}=\frac{1}{2}\left(1-\xi_{\mathbf{k}} / E_{\mathbf{k}}\right)
$$

and

$$
\left|v_{\mathbf{k}}\right|^{2}=\frac{1}{2}\left(1+\xi_{\mathbf{k}} / E_{\mathbf{k}}\right) .
$$

The quasiparticle energy $E_{\mathbf{k}}=\left(\xi_{\mathbf{k}}^{2}+d_{\mathbf{k}}^{2}\right)^{1 / 2}$, where

$$
\xi_{\mathrm{k}}=\left(\cos k_{x}+\cos k_{y}\right) \cos (\pi / 2) \Phi
$$

and

$$
d_{\mathrm{k}}=-\left(\cos k_{x}-\cos k_{y}\right) \sin (\pi / 2) \Phi .
$$

Now the trial wave function for SFP can be constructed,

$$
|\psi\rangle=P_{d} \prod_{k<k_{F}} \tilde{C}_{-\mathbf{k} \uparrow}^{\dagger} \tilde{C}_{\mathbf{k} \downarrow}^{\dagger}|0\rangle,
$$

where $k_{F}$ represents the Fermi surface and is determined by the number of particles present.

There is only one variational parameter $\Phi$ in the SFP trial wave function. This is similar to the RVB wave function $^{4,5}$ where the bond strength or the gap $\Delta$ is the only variational parameter. At half filling, because of SU(2) symmetry, the two wave functions are, in fact, the same ${ }^{7}$ with the identification ${ }^{4} \Delta=2 \tan (\pi / 2) \Phi$. We have calculated the magnetic energy per site for several values of $\Phi$ as shown by the open squares in Fig. 1. The dashed line is

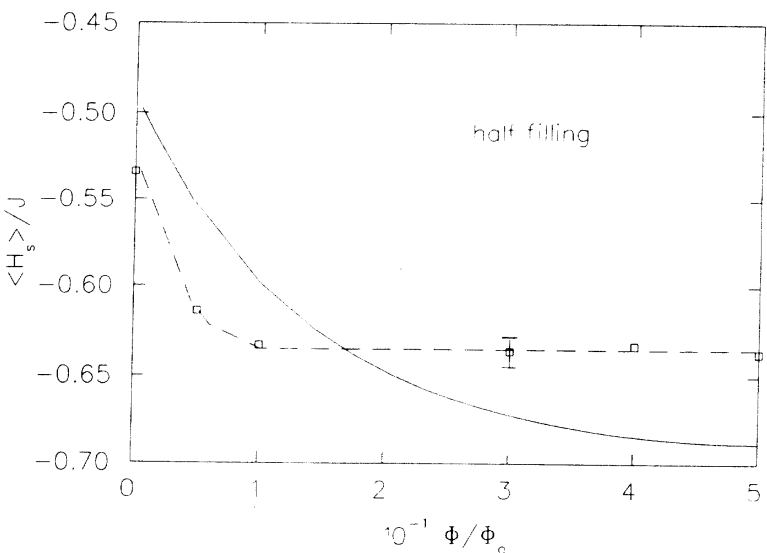

FIG. 1. Magnetic energy per site $\left\langle H_{s}\right\rangle / J$ as a function of the magnitude of staggered flux $\Phi$ at half filling. Open squares are the numerical result for a lattice of 82 sites, and the solid line is the result using Gutzwiller approximation. The dashed line is a guide for the eye.

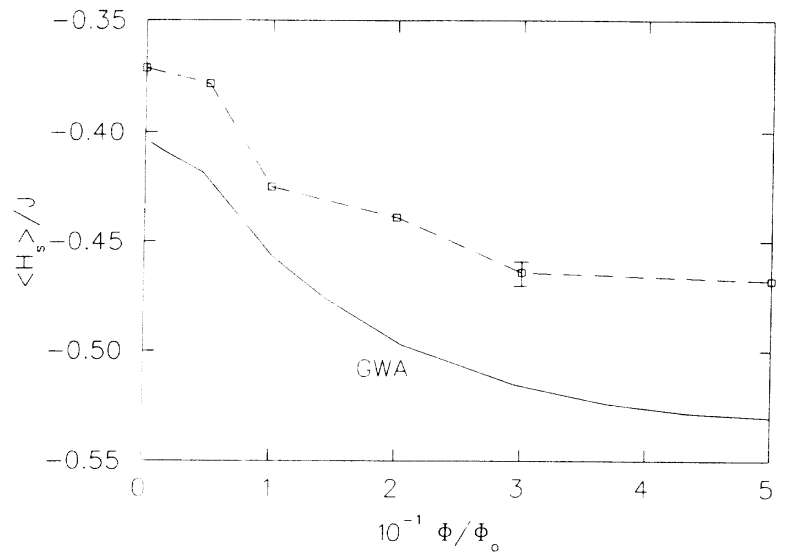

FIG. 2. Same as Fig. 1, except now there is a finite hole concentration $\delta=\frac{8}{82}$.

a guide for the eye. It is quite surprising to find that within the numerical accuracy, the energy is independent of $\Phi$ unless $\Phi<0.1$. It should be noted that the equivalence of RVB states and SFP states also have been verified numerically. In a semilogarithmic plot it would seem there is a minimum as shown in Ref. 4 . We believe that the local time-reversal symmetry should not be broken at half filling because there is only the Heisenberg interaction. Hence the SFP wave function has the right symmetry for $\left\langle H_{s}\right\rangle$ to be independent of $\Phi$.

We have also calculated the energy analytically by using the Gutzwiller approximation (GWA). In this approximation the SFP and RVB are again identical at half filling. The details of the GWA for the RVB state can be found in Ref. 4. The result of GWA is plotted as the solid line in Fig. 1. Although the GWA produces the correct lowest-energy state $\left(\Phi=\frac{1}{2}\right)$ that preserves time-reversal symmetry, it cannot account for the $\Phi$ independence observed in the numerical calculations of $\left\langle\boldsymbol{H}_{\boldsymbol{s}}\right\rangle$.

Away from half filling, the kinetic-energy term $H_{t}$ becomes very important for $t \gg J$. The average magnetic energy $\left\langle\boldsymbol{H}_{s}\right\rangle$ and kinetic energy $\left\langle\boldsymbol{H}_{t}\right\rangle$ are plotted (the open squares) as a function of staggered flux $\Phi$ in Figs. 2 and 3, respectively. The hole concentration is $\delta=\frac{8}{82} \simeq 0.1$. As expected, the kinetic energy favors zero flux while the

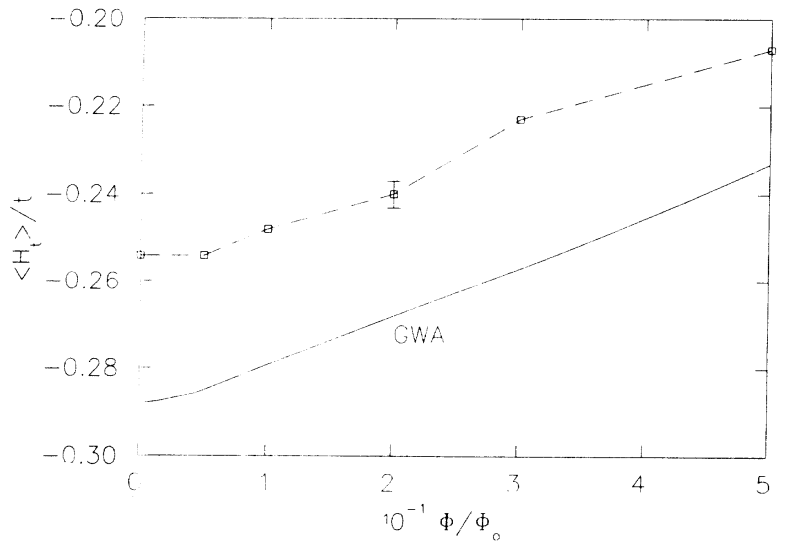

FIG. 3. The kinetic energy as a function of $\Phi$ for hole concentration $\delta=\frac{8}{82}$. Symbols have the same meaning as in Fig. 1 . 


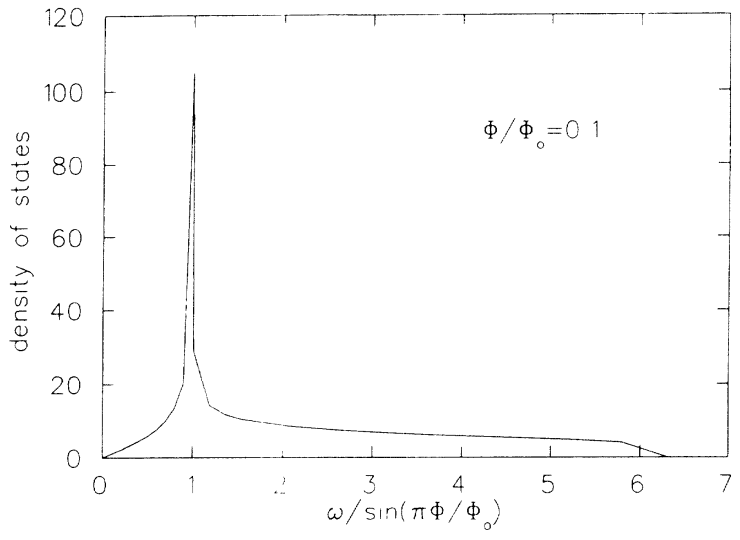

FIG. 4. Density of states as a function for the quasiparticle of energy $E_{k}$ in the SFP. The magnitude of flux is 0.1 .

magnetic energy favors $\Phi=\frac{1}{2}$. What is not expected is the sharp rise of $\left\langle H_{s}\right\rangle$ near $\Phi_{c} \simeq 0.08$. Because of this sharp rise, the state with the lowest total energy $\left\langle H_{s}\right\rangle+\left\langle H_{t}\right\rangle$ for $2 \leq t / J \leq 6$ is around $\Phi_{c}$. To understand this result further, we have made an analytical calculation using the GWA (Ref. 10) with the Gutzwiller projection operator $P_{d}$ replaced by the geometric factors $g_{t}=2 \delta /$ $(1+\delta)$ and $g_{s}=4 /(1+\delta)^{2}$ for the kinetic and magnetic energies, respectively. The results are plotted as the solid line in Figs. 2 and 3. The numerical result, which takes into account the projection operator exactly, seems to have more sharp features, such as steps and plateaus, then the smooth variation of the result of the GWA, but the rapid increase of $\left\langle H_{s}\right\rangle$ near $\Phi_{c}$ is still observed. The significance of $\Phi_{c}$ is better understood by examining the density of states of quasiparticle energy $E_{k}$ of the SFP state. The density of states plotted in Fig. 4 for $\Phi=0.1$ shows a very strong Van Hove singularity with logarithmic divergence. For this particular value of energy, the equal-energy surface in the Brillouin zone is nested and the nesting wave vectors are $Q_{n}=\pi[1-\Phi, \pm(1-\Phi)]$. For a fixed concentration of holes, as the staggered flux $\Phi$ decreases from $\frac{1}{2}$ to 0 , the Van Hove singularity passes through the Fermi surface at $\Phi_{c}=1-\sqrt{1-\delta}$ and the magnetic energy increases sharply.

For hole concentration $\delta \simeq 0.2$, the magnetic energy becomes relatively unimportant. The projected Fermi-liquid state, $\Phi=0$, has the lowest total energy for $t / J>2$. In Tables I and II, where $\delta \simeq 0.1$ and 0.2 , respectively, total energies of the $\Phi=\frac{1}{2}$ flux phase, $\Phi=0$ state, the $d$-wave RVB state, and the SFP state at $\Phi=0.1$ are compared for $t / J=2$ and 5. Notice that the $\Phi=\frac{1}{2}$ state is also the lowest energy state of all the CFP states ${ }^{12}$ for $\delta<0.2$ and $t / J=2$. Hence for physically interesting parameter values

TABLE I. Total energies for four different states are compared. The hole concentration is $\delta=\frac{8}{82}$.

\begin{tabular}{lcccc}
\hline \hline$\langle H\rangle / J$ & $\Phi=\frac{1}{2}$ & $\Phi=0$ & RVB & $\Phi=0.1$ \\
\hline$t / J=2$ & $-0.882(3)$ & $-0.879(5)$ & $-0.961(6)$ & $-0.921(4)$ \\
$t / J=5$ & $-1.503(4)$ & $-1.641(8)$ & $-1.692(10)$ & $-1.665(7)$ \\
\hline \hline
\end{tabular}

TABLE II. Same as Table I, except $\delta=\frac{16}{82}$.

\begin{tabular}{lcccc}
\hline \hline$\langle H\rangle / J$ & $\Phi=\frac{1}{2}$ & $\Phi=0$ & $\mathrm{RVB}^{\mathrm{a}}$ & $\Phi=0.1$ \\
\hline$t / J=2$ & $-1.009(4)$ & $-1.250(4)$ & $-1.250(7)$ & $-1.238(4)$ \\
$t / J=5$ & $-2.008(7)$ & $-2.875(7)$ & $-2.87(2)$ & $-2.642(7)$ \\
\hline \hline
\end{tabular}

a Numbers in this column are obtained from Ref. 4.

CFP seems to be unfavorable. For large doping (20\% or more), $\Phi=0$ or the projected Fermi-liquid state has the lowest energy. Even the $d$-wave superconducting RVB state is not superior. But for smaller doping concentration, SFP seems to be quite favorable as the normal state.

Since the $d$-wave RVB state has lower energy than the SFP, it then becomes interesting to find out if there is Cooper-pairing instability for the SFP. Recently Zhang ${ }^{10}$ demonstrated this instability by using the GWA. Here we shall examine this instability by directly evaluating the energy of a BCS state formed by the quasiparticles of the SFP. The wave function is of the form

$$
\left|\psi^{\prime}\right\rangle=P_{d} \prod_{k}\left(\alpha_{k}+\beta_{k} \tilde{C}_{\mathbf{k}\rangle}^{\dagger} \tilde{C}_{-\mathbf{k} \downarrow}^{\dagger}\right)|0\rangle,
$$

where

$$
\begin{gathered}
\left|\alpha_{\mathbf{k}}\right|^{2}=\frac{1}{2}\left(1-\frac{E_{k}-\mu}{\epsilon_{k}}\right),\left|\beta_{\mathbf{k}}\right|^{2}=\frac{1}{2}\left(1+\frac{E_{k}-\mu}{\epsilon_{k}}\right), \\
\alpha_{k} \beta_{k}^{*}=\frac{\Delta_{k}}{2 \epsilon_{k}}
\end{gathered}
$$

and

$$
\epsilon_{k}=\left[\left(E_{k}-\mu\right)^{2}+\Delta_{k}^{2}\right]^{1 / 2} .
$$

Two different forms of the gap parameter $\Delta_{k}$ have been chosen: $\Delta_{k}=\Delta$ for the $s$ wave and $\Delta_{k}=\Delta\left(\cos k_{x}-\cos k_{y}\right)$ for the $d$ wave. In the several cases we have studied where $\Delta=0.1$ and 0.3 , the energies of the superconducting SFP state $\left|\psi^{\prime}\right\rangle$ is always higher than the simple SFP state. In this simple calculation only the lower quasiparticle band in the SFP state is used and we do not find superconducting instability. On the other hand, if we use a more sophisticated wave function obtained by Zhang ${ }^{10}$ that includes both quasiparticle bands, ${ }^{13}$ the $d$-wave superconducting instability is observed. But at $10 \%$ doping we found pure $d$ wave has the lowest energy and the superconducting state maintains time-reversal symmetry.

In summary, we have presented numerical results of variational energies calculated for SFP, for $10 \%$ hole concentration and $2 \geq t / J \geq 6$. SFP, with a nested Fermi surface, has lower energy than the projected Fermi liquid. For $20 \%$ doping, the Fermi liquid would be more favorable. We also have not found any evidence for coexistence of superconductivity and SFP in the hole concentration range of $10 \%$ to $20 \%$.

In concluding this paper we wish to point out that when fluctuations are considered, the projected Fermi-liquid state could be more favorable than the SFP even at a mere $10 \%$ doping. But the competitiveness between SFP and the projected Fermi liquid indicates the importance of the flux variables. This would support the recent work of Nagaosa and Lee. ${ }^{14}$ 
This work was partially supported by the Thomas F. Jeffress and Kate Miller Jeffress Memorial Trust, and by U.S. Department of Energy Contract No. DE-AS05-80ER 10713.

${ }^{1}$ P. W. Anderson, Science 235, 1196 (1987).

${ }^{2}$ F. C. Zhang and T. M. Rice, Phys. Rev. B 37, 3759 (1988).

${ }^{3}$ P. W. Anderson, G. Baskaran, Z. Zou, and T. Hsu, Phys. Rev. Lett. 58, 2790 (1987).

${ }^{4}$ F. C. Zhang, C. Gros, T. M. Rice, and H. Shiba, Supercond. Sci. Technol. 1, 36 (1988).

${ }^{5}$ S. Liang, B. Doucot, and P. W. Anderson, Phys. Rev. Lett. 61, 365 (1988); T. K. Lee and Shiping Feng, Phys. Rev. B 38, 11809 (1988).

${ }^{6}$ I. Affleck and J. B. Marston, Phys. Rev. B 37, 3774 (1988).

${ }^{7}$ P. W. Anderson, B. S. Shastry, and D. Hristopulos, Phys. Rev. B 40, 8939 (1988); D. Poilblanc, (unpublished).

${ }^{8}$ D. Poilblanc, Y. Hasegawa, and T. M. Rice, Phys. Rev. B 41,
1949 (1990); D. Poilblanc and Y. Hasegawa, ibid. 41, 6899 (1990); P. Lederer, D. Poilblanc, and T. M. Rice, Phys. Rev. Lett. 63, 1519 (1989).

${ }^{9}$ A. Fetter, C. Hanna, and R. Laughlin, Phys. Rev. B 39, 9679 (1989); Y. H. Chen, F. Wilczek, E. Witten, and B. Halperin, Int. J. Mod. Phys. B 3, 1001 (1989).

${ }^{10}$ F. C. Zhang, Phys. Rev. Lett. 64, 974 (1990).

${ }^{11}$ T. Dombre and G. Kotliar (unpublished).

${ }^{12}$ Shoudan Liang and Nandini Trivedi, Phys. Rev. Lett. 64, 232 (1990)

${ }^{13}$ This point is brought to our attention by F. C. Zhang (private communication).

${ }^{14}$ N. Nagaosa and P. A. Lee, Phys. Rev. Lett. 64, 2450 (1990). 